\title{
Las TIC en la enseñanza de los negocios internacionales Un asunto de competencias comunicativas
}

\author{
ÁNGELA JULIETA MORA RAMÍREZ \\ Fecha de recepción: 11-06-2009 - Aprobación: 19-06-2009
}

\section{Resumen}

Este artículo es una descripción sobre la utilización de las TIC en la enseñanza de los negocios internacionales, desde las competencias comunicativas, documento, que permite identificar la apropiación de este tipo de competencias en los estudiantes a partir de los primeros semestres de Negocios Internacionales.

\section{Palabras claves}

TIC, competencias, comunicación, Negocios Internacionales.

\begin{abstract}
This article is a brief description on the use of Iст in teachinginternationalbusiness, fromcommunication skills, establishing a paper that allows to identify the ownership of such skills in students from the first semesters of International Business.
\end{abstract}

\section{Keywords}

ICT, competences, communication, international business

\section{Las tecnologías de la información}

Las tecnologías de la información y comunicaciones TIC, han tomado una fuerza importante, en la última década, y se han generalizado hacia todas las áreas del conocimiento.

Una definición, muy acertada, y concreta de las Tic, es la realizada por la Unión Europea (2009):

\section{Las tecnologías de la información y de las comunicaciones (TIC), son un término que se utiliza actualmente para hacer referencia a una gama amplia de servicios, aplicaciones, y tecnologías, que utilizan diversos tipos de equipos y de programas informáticos, y que a menudo se transmiten a través de las redes de telecomunicaciones.}

Frente a tan importante herramienta, el objetivo de este artículo es evidenciar, y poner en conocimiento de los estudiantes y compañeros docentes, de primeros semestres, de Negocios Internacionales de la universidad Politécnico Grancolombiano, un instrumento clave y moderno, que implica establecer mecanismos, para que los estudiantes, se apropien de las tecnologías y las utilicen como ayudas sólidas, de negocios y de aplicación general, especialmente, en un mundo cada vez más globalizado e interdependiente.

Las opciones digitales, el correo, y la inmediatez de los negocios en línea generan, estrategias de comunicación dinámicas a velocidades altas; así se pueden cerrar y concretar grandes negocios. Sin la necesidad de desplazarse y acercan de manera directa al mundo de hoy globalizado e interdependiente. 


\section{ÁNGELA JULIETA MORA RAMÍREZ.}

Economista de la Universidad Pedagógica y Tecnológica (Tunja), especialista en Integración en el Sistema Internacional. Es investigadora y docente, desde hace más de diez años, fundadora de la revista electrónica Expectativas de la Universidad Jorge Tadeo Lozano, editora y gestora de revistas web, escribe para revistas de divulgación e indexadas, nacionales e internacionales, sobre temas pedagógicos y de su área específica de conocimiento.

Es líder del grupo de la Facultad de Ciencias Administrativas y Económicas del Politécnico Grancolombiano y SU GRUPLAC, árbitro y par de revistas indexadas nacionales. Actualmente cursa la maestría en Educación con Énfasis en Investigación y es directora del Centro de Investigación "Colombia Regional y Empresarial" del Politécnico Grancolombiano.

julietamora22@hotmail.com

amoraram@poligran.edu.co

\section{La educación de Negocios Internacionales y las TIC}

Cada vez más, nos asombramos con los adelantos tecnológicos. La realidad se hace más dinámica, los contenidos, y flujos de información se masifican, en especial para la comunidad académica, aportando competencias de comunicación acordes con los insumos diseñados por el mundo digital actual.

Según María Stella Girón y Marco Antonio Vallejo (1992)

La competencia comunicativa comprende las aptitudes y los conocimientos que un individuo debe tener para poder utilizar sistemas lingüísticos y translingüísticos que están a su disposición para comunicarse como miembro de una comunidad sociocultural dada.

Estas competencias no solo se realizan por parte del estudiante futuro negociador, se efectúan, durante el proceso académico en el aula, en el desarrollo de la investigación formativa, de tal manera que el estudiante desde el primer semestre pueda participar en mesas de negociación simuladas digitalmente.

Estos procesos prácticos son parte de la estrategia de los proyectos de aula de los primeros semestresy demuestran la vinculación y acercamiento entre estudiante y docente, en función de la aplicación de las competencias y el desarrollo de habilidades comunicativas, tan esenciales en el mundo digital.

La utilización de las tecnologías nuevas, y vinculación en el aula de las TIC, es indispensable en la nueva educación, que requiere estudiantes más globales, que se adapten de manera más rápida a la dinámica actual del mundo. Según Marqués Graells (2000)

Las Tic (informática, telemática, multimedia...) nos facilitan la realización de nuestros trabajos porque, sean éstos los que sean, siempre requieren cierta información para realizarlo, un determinado proceso de datos y a menudo también la comunicación con otras personas; y esto es precisamente lo que nos ofrecen las Tic:

- Acceso a todo tipo de información.

- Todo tipo de proceso de datos, y de manera rápida y fiable.

- Canales de comunicación inmediata, sincrónica y asincrónica, para difundir información y contactar cualquier persona o institución del mundo.

Es importante que estas herramientas se conviertan, en parte integral del aula, donde la estrategia pedagógica y receptiva, sea siempre visualizar de qué manera, la tecnología sirve como instrumento de capacitación y de aprendizaje continuo.

Así mismo es de resaltar que el acceso no sea limitado y se amplíe a cada área del conocimiento de su línea específica profesional. No se debe olvidar que hoy en día todo se hace vía digital.

Existen grandes formas de ver el mundo, por medio de un manejo responsable de los medios y de las comunicaciones se pueden hacer negocios por internet, viajes, amigos, y hasta conquistas... Pero hay que ser responsables de su manejo y uso, debemos conocer y capacitarnos, pues quien no maneje la tecnología se encuentra obsoleto.

Los cambios generan más dinámicas, más interacciones, y formas de hacer las cosas. Es importante entonces establecer puntos de estudio y de soporte para no quedarnos atrás e involucrar de manera continua estos recursos con el fin de hacer parte del mundo digitalizado y descubrir lo bueno de la internet.

De acuerdo con el profesor Marques (2000):

Las grandes funcionalidades de las Tic como fuente de información (acceso a todo tipo de bases de datos, información puntual de los eventos científicos de todo el mundo), 
canal de comunicación e instrumento para el proceso de datos, facilitan enormemente muchas labores investigadoras y abren nuevas posibilidades de colaboración "a distancia" con especialistas de todo el mundo.

Además, la publicación de trabajos en internet aumenta la transparencia de la labor que se realiza en cada centro investigador y permite la rápida difusión y (sic) compartición del saber.

Por otra parte, la veloz dinámica de la sociedad de la información le confiere una gran avidez por la investigación, de la que necesita datos para tomar decisiones acertadas y seguir progresando. Esta circunstancia favorable al crecimiento de la investigación en general, juntamente con el hecho de que mediante los servicios telemáticos las universidades y las empresas pueden conocer bien lo que hacen, lo que investigan, lo que necesitan, y también pueden comunicarse con facilidad, explica el aumento de colaboración entre ellas.

Es importante, apropiarnos de la tecnología, y generar cambios que puedan dinamizar nuestra creatividad, hacer que tanto estudiantes como docentes, implementemos formas de conocer, y divulgar a través de la tecnología, asuntos importantes, negociaciones en línea, y acuerdos recientes, de países y de empresas.

Es evidente que se tiene a mayor alcance la información, pero, como su acceso es tan fácil, no podemos caer en la trampa, del copy-paste, ni del facilismo; se debe entonces ampliar la visión exploratoria, y aprender a pensar, seleccionar los mejores autores, las mejores páginas, buscar agilidad en procesos mediáticos, como un negocio internacional.

La utilización de las tecnologías de las comunicaciones y la información, son instrumento estructural en el desarrollo de los jóvenes de hoy, el aprovechamiento de estas tecnologías, es motivador pues los estudiantes viven en función del ipod, de la internet, del chat y de todo lo que tenga que ver con herramientas tecnológicas.

Por tanto, la educación moderna debe conciliar con estos avances para potencializar las competencias comunicativas y tecnológicas propias de la generación moderna, el Programa de Negocios del Departamento de Economía del Politécnico Grancolombiano no es ajeno a estos cambios. Entonces es el momento de avanzar y fortalecer, buscar alternativas donde el insumo sea la tecnología y el aprovechamiento de las competencias comunicacionales.
Según Fredy Palacino Rodríguez (2007):

Desde que Hymes (1984 citado en Lomas, 2006) recontextualizó el sentido de la competencia lingüística, reconoció la orientación social de la competencia y resaltó en ella el valor de la comunicación (competencia comunicativa), que existe entre las personas que aprenden y construyen conocimiento en las diferentes situaciones comunicativas que se presentan dentro de las sociedades.

El desarrollo de estudio de casos virtuales y juegos de simulación, en los que interviene la tecnología, es un insumo que desde primer semestre se debe buscar, de tal forma que se construya desde la hermenéutica, enseñanza sincrónica y asincrónica de ciudadanos del mundo y para el mundo.

\section{Conclusiones}

Ser profesional en Negocios Internacionales requiere ampliar la proyección y la información, requiere datos reales, que nos proporcionan las nuevas tic. Por tanto es esencial, que los estudiantes de primeros semestres de este programa, empecemos a repensar la enseñanza de los Negocios Internacionales.

Ser parte de un mundo globalizado, implica buscar ventajas sobre la tecnología actual, no relegarse de lo nuevo, estar a la vanguardia de la educación moderna, más aun en el área de los Negocios Internacionales que requieren visualización, hacerse partícipes dinámicos de este mundo cambiante, cada vez más globalizado y ágil.

Se trata de manejar esta tecnología para beneficio propio, en función del crecimiento académico continuado, el cual debe ser premisa pedagógica de la enseñanza en Negocios Internacionales, buscar una continua retroalimentación, crear, conocer y descubrir. Es preciso ampliar las competencias comunicativas en lo estudiantes y desarrollar estrategias de sincronía a favor de la negociación, como medio y como fin.

Las áreas internacionales han desarrollado miles de estrategias para complementar las competencias, desde la estrategia de la negociación digital apoyo a los estudiantes, en el desarrollo, socialización, ejecución de juegos de simulación, negociadora, nuestra institución abandera el proceso desde el Departamento de Economía, la Facultad de Ciencias Administrativas y Económicas, generando trasnversalidad y apropiación de las Tic, en función de la educación y la consolidación de las competencias comunicativas de los estudiantes de primeros semestres. 
La utilización de las TIC es necesaria en toda carrera. Los negocios internacionales requieren de estas tecnologías en su aplicación y direccionamiento hacia una educación moderna, y basada en las ventajas de internet, específicamente en busca de negocios nuevos y en la estructuración formal entre educación y elementos digitales, instrumentos fundamentales en la interdependencia económica actual.

\section{BIBLIOGRAFÍA}

Girón, M. S.; Vallejo, M.A. (1992). La competencia comunicativa. Universidad de Antioquia. Obtenido el 19 de junio de 2009, de http://docencia.udea.edu.co/LenguaMaterna/ Documentos/UNIDAD\%2013.doc

Marqués, G. P. (2000). Impacto de las TICs, en la enseñanza universitaria. Última versión. Departamento de Pedagogía Aplicada. Universidad de Barcelona. Obtenido el 24 de abril de 2008, de http://dewey.uab.es/PMARQUES/ticuniv. htm\#inicio

Mora, A. J. (2003). “La Globalización del Caos". Revista Expectativas (en línea) www.utadeo.edu.co/expectativas

Mora, A.J. (2008.b). El constructivismo y la educación en la CAN, Un punto de vista alternativo. Revista Debates Latinoamericanos, Centro Latinoamericano de Investigación de Estudios Avanzados, Obtenido el 11 de junio de 2009, de www.clea.ar

Palacino, Rodríguez, F. (2007). Competencias comunicativas, aprendizaje y enseñanza de las Ciencias Naturales: un enfoquelúdico. RevistaElectrónicadeEnseñanzadelasCiencias. Obtenidoel 19 de junio de 2009, de http://74.125.47.132/ search?q=cache:ExyCN2CbSQJ:reec.uvigo.es/volumenes/volumen6/ART4_Vol6_N2.pdf+competencias+comunic ativas\&cd $=59 \& \mathrm{hl}=\mathrm{es} \& \mathrm{ct}=\mathrm{clnk} \& \mathrm{gl}=$ consultado

Unión Europea. (2005). Tecnologías de la información y de la comunicación en el ámbito del desarrollo - El papel de las TIC en la política comunitaria de desarrollo. Eur - Lex (En línea) http://209.85.207.104/ search?q=cache:jlmHItUKLrMJ:www.ecomaccess.com/ iim/docs/ict_es.doc+definicion+TICs\&hl=es 